\title{
Shigella flexneri Sepsis in a Well-nourished Immunocompetent Child: A Rare Case Report
}

\author{
Rama Gupta ${ }^{1}$, Veenu Gupta ${ }^{2}$, Deepak Bhat ${ }^{3}$, Reetu Goyal ${ }^{4}$, Jyoti Chaudhary ${ }^{5}$, Menal Gupta ${ }^{6}$
}

\begin{abstract}
Shigella infections are usually restricted to the intestine. There are a few reports of Shigella isolated from the blood and most of these are from children, usually the neonates and the malnourished. In the small number of adult cases of Shigella bacteremia which have been reported, there appears to be an association with underlying disease and immunosuppression including acquired immunodeficiency syndrome. We report a rare case of septicemia with Shigella flexneri in a well-nourished, obese child, with no other predisposing underlying condition. With the prompt and aggressive symptomatic treatment along with administration of appropriate antibiotics based upon the antimicrobial susceptibility pattern of the isolate, the patient's recovery was uneventful.
\end{abstract}

Keywords: Bacteremia, Intravenous antibiotics, Shigella flexneri, Stool culture, Well-nourished child.

Journal of Gastrointestinal Infections (2021): 10.5005/jp-journals-10068-3046

\section{INTRODUCTION}

Shigellosis remains an important public health problem in developing and underdeveloped countries, including India. The disease is transmitted through feco-oral route due to consumption of contaminated potable water. There are four important species of Shigella, i.e., Shigella dysenteriae, S. flexneri, S. boydii, and S. sonnei, also known as groups $A, B, C$, and $D$, respectively. 'The spectrum of the disease varies from mild gastrointestinal (GI) symptoms to severe and fatal septicemia/septic shock, though rare. ${ }^{2,3}$ The primary Gl symptoms include acute bloody diarrhea and fever, which are enterotoxin mediated. ${ }^{4}$ Other extraintestinal organ system which may rarely get involved, include urinary tract, joints, liver, and central nervous system especially in young malnourished children. In most of the cases disease is self-limited or requires only supportive care, and symptoms resolve within 1 week. ${ }^{5,6}$

Shigella bacteremia seems to be a rare complication of GI shigellosis and is usually associated with underlying disease like malnutrition. Other predisposing conditions which have been associated include age, diabetes, leukemia, sickle cell anemia, malignancy, cirrhosis, immunosuppression, and HIV infection..$^{7-9}$

This is a rare case report of shigellosis leading to bacteremia in a well-nourished, obese child, who did not have any other underlying condition, except for her young age.

\section{Case Description}

A5-year-old female child from Ludhiana admitted to a referral hospital during August, 2021, presented with multiple episodes of watery diarrhea and high-grade fever since the past 4 days. In addition, the patient complained of abdominal pain for the last 2 days. On physical examination, the liver and the spleen were not palpable; abdomen was soft, tender, nondistended, with the presence of tenesmus and bowel sounds. The heart rate was 124/minute, respiratory rate $23 /$ minute, blood pressure was $100 / 70 \mathrm{~mm} \mathrm{Hg}$, and body temperature was $98.6^{\circ} \mathrm{F}$. Anthropometric details of the child were weight $27 \mathrm{~kg}$ (expected weight-16 kg), length $108 \mathrm{~cm}$ (expected-109 cm) and BMI 23 (expected-13). On auscultation, bilateral air entry was observed with no crepitations/ronchi.
1,2,4-6 Department of Microbiology, Dayanand Medical College and Hospital, Ludhiana, Punjab, India

${ }^{3}$ Department of Pediatrics, Dayanand Medical College and Hospital, Ludhiana, Punjab, India

Corresponding Author: Veenu Gupta, Department of Microbiology, Dayanand Medical College and Hospital, Ludhiana, Punjab, India, Phone: +919814736051, e-mail: vsunilgupta@rediffmail.com

How to cite this article: Gupta R, Gupta V, Bhat D, et al. Shigella flexneri Sepsis in a Well-nourished Immunocompetent Child: A Rare Case Report. J Gastrointest Infect 2021;11:44-46.

Source of support: Nil

Conflict of interest: None

CVS-S1S2 was normal without any murmur. CNS-E4V5M6, bilateral pupil ESRL, tone/power normal, no signs of cranial nerve palsy/ meningeal irritation.

The child was allergic to ceftriaxone and metronidazole. Provisional diagnosis of acute dysentery with obesity was made and the child was managed as a case of acute dysentery and treated with intravenous antibiotics (amikacin and ciprofloxacin) along with intravenous fluids and other supportive care. The stool routine examination showed white blood cells 10-15/HPF with 2-3 red blood cells/HPF. Ultrasound abdomen was suggestive of mild splenomegaly, and thickened large gut bowel in left hemi-abdomen, suggestive of infective/inflammatory etiopathogenesis.

Complete blood count reported hemoglobin $10.8 \mathrm{mg} / \mathrm{dL}$, total leukocyte count: $8700 / \mathrm{cu} \mathrm{mm}$ (neutophils $40.7 \%$; lymphocytes $45.8 \%$; monocytes $11.3 \%$; eosinophils $02 \%$, and basophils $0 \%$ ) with a platelet count of $320 \times 10^{3} / \mu \mathrm{L}$. No parasites were observed in the blood film. Liver function tests revealed total bilirubin of $0.38 \mathrm{mg} / \mathrm{dL}$ with all other tests being normal. Renal functions parameters were normal and C-reactive protein was 34.1.

Considering septic shock as a possibility, blood culture and other relevant investigations were requested. Blood culture was performed using BACTEC 9120 and identification and susceptibility testing was done by Vitek 2. Blood culture was positive for 
S. flexneri. The isolate was sensitive to cotrimoxazole (MIC $\leq 20 \mu \mathrm{g} / \mathrm{mL}$ ), cefipime (MIC $\leq 0.12 \mu \mathrm{g} / \mathrm{mL}$ ), ceftriaxone (MIC $\leq 0.12 \mu \mathrm{g} / \mathrm{mL}$ ), azithromycin, and resistant to amikacin (MIC $4 \mu \mathrm{g} / \mathrm{mL}$ ), gentamicin (MIC $4 \mu \mathrm{g} / \mathrm{mL}$ ), ciprofloxacin (MIC $>4 \mu \mathrm{g} / \mathrm{mL}$ ), levofloxacin (MIC $>8 \mu \mathrm{g} / \mathrm{mL}$ ). Susceptibility to azithromycin was done by Kirby Bauer disk diffusion test. Based upon the antibiotic susceptibility pattern, azithromycin was added to the treatment regimen. Additionally, a request for stool culture was also sent after the blood culture turned out to be positive for S. flexneri. Culture of stool was done on MacConkey agar and deoxycholate citrate agar which grew nonlactose fermenting colonies after incubation at $37^{\circ} \mathrm{C}$ for 24 hours and was identified using automated identification system (Vitek 2 compact, BioMerieux, place) as S. flexneri and further confirmed by serotyping.

The general condition of the child improved gradually and was discharged in a stable condition after 8 days of hospital stay.

\section{Discussion}

Shigellosis is primarily caused by S. dysenteriae, S. flexneri, S. boydii, and S. sonnei. S. dysenteriae type I, and S. flexneri are among the most virulent serotypes, associated with invasive shigellosis leading to septicemia. ${ }^{10,11}$ Though an invasive disease, it usually does not invade the tissue beyond the lamina propria and hence infrequently cause bacteremia in some patients with predisposing conditions. These predisposing conditions include young age, malnutrition, and immune-suppression. ${ }^{12}$ A case report of $S$. sonnei septicemia with enterocolitis in a newborn infant had been documented..$^{13}$ Authors had emphasized that septicemia with enterocolitis was responsible for exudative loss of immunoglobulins, complement, and various plasma proteins which resulted in compromised immune response in the form of impaired lysis and opsonization of invading bacilli leading to devastating sepsis.

A study from Argentina has described two cases of $S$. flexneri bacteremia in infants. ${ }^{14}$ Both the patients presented with fever and diarrhea. One of them had primary immune deficiency, whereas the other patient had no other underlying predisposing condition. Another study from Mumbai has reported four cases of Shigella septicemia in children admitted to Lokmanya Tilak Municipal General Hospital. Out of these four shigellosis cases, three were caused by S. dysenteriae serotype I and one by S. flexneri; a mortality rate of $75 \%$ was reported. Probable reason for high mortality with Shigella septicemia in these patients could be multidrug resistant Shigella species and a majority of them had S. dysenteriae type I infection, which is most virulent of all the serotypes of Shigella. All these isolates were sensitive to gentamicin, amikacin, norfloxacin, and nalidixic acid but resistant to amoxicillin, chloramphenicol, tetracyclin, and cotrimoxazole. ${ }^{11}$ However, another meta-analysis of Shigella isolates had reported $20 \%$ resistance to nalidixic acid increasing levels of resistance to third-generation cephalosporins and azithromycin. ${ }^{15}$ On the other hand, the present isolate was susceptible to azithromycin. Another rare case of fatal septicemia due to multidrug-resistant S. flexneri foregoing an episode of $\mathrm{Gl}$ infection has also been reported in a 6-month-old infant from New Delhi. ${ }^{5}$ It was an exceptional presentation of Shigella septicemia with severe shock, disseminated intravenous coagulation, and convulsions.

Although shigellemia is primarily reported from malnourished infants and children, there are a few reports where adult population was also reported to manifest bacteremia due to different species of Shigella. Sharma and Arora reported an uncommon case of
S. flexneri bacteremia in a 65-year-old nondiabetic, hypertensive male with a history of dysentery. The patient from New Delhi was a known case of chronic kidney disease, dilated cardiomyopathy, and permanent pacemaker device implanted since the past 7 years. ${ }^{12}$ Another series of case reports have documented three adult cases in which Shigella was isolated from the blood. Two of these patients made an uneventful recovery while the third died. An underlying cause of immunosuppression was suspected in this patient but unproven. ${ }^{9}$

Though shigellosis results in considerable morbidity in endemic areas, mortality is rare in developed countries. However, a study from Israel has reviewed all pediatric (age 5 months to 11 years) deaths $(n=15)$ following shigellosis during a span of 10 years. Predominantly, S. flexneri was the concerned Shigella species in these patients $(n=8)$ followed by S. sonnei $(n=4)$ and S. dysenteriae $(n=1)$. However, two of the Shigella species could not be identified. The cause of death in all these patients was unswerving with noxious encephalopathy, except for one case where "Reye-like" syndrome was found to be associated with the death of the patient. Case-control study of these patients with the surviving shigellosis hospitalized patients was found to have similar incidence of fever, diarrhea, vomiting, dehydration and convulsions, and toxic encephalopathy due to childhood shigellosis in a developed country. ${ }^{16}$

In the present case report, septicemia was caused by S. flexneri, as isolated from both the blood and the stool samples. Additionally, the isolate was susceptible to many antibiotics and the patient was treated symptomatically along with azithromycin. The patient was discharged in a stable condition and recovery was uneventful after a stay of 8 days in the hospital.

\section{Conclusion}

The present study emphasizes the importance of considering Shigella infection as a differential diagnosis when a patient with severe sepsis associated with diarrhea and vomiting is encountered. The possibility of shigellemia should be considered not only in malnourished/immunocompromised children but also in well-nourished/immunocompetent patients presenting with acute febrile gastroenteritis. The patient should be aggressively treated as the disease may progress very rapidly. Further, antibiotic susceptibility profile of the isolate should be kept in mind while treating such patients especially when the patient does not respond even after 48 hours of empirical antibiotic treatment, which could be life-saving.

\section{References}

1. Levine $M M$, Kotloff $K L$, Barry EM, et al. Clinical trials of Shigella vaccines: two steps forward and one step back on a long, hard road. Nat Rev Microbiol 2007;5(7):540-553. DOI: 10.1038/nrmicro1662.

2. Niyogi SK. Shigellosis. J Microbiol 2005;43(2):133-143. PMID: 15880088.

3. Erqou SA, Teferra E, Mulu A, et al. A case of shigellosis with intractable septic shock and convulsions. Jpn J Infect Dis 2007;60(5):314. PMID: 17881877.

4. Goldberg M, Calderwood SB, Edwards MS, et al. Shigella infection: epidemiology, microbiology, and pathogenesis. Recuperado el 2013;14:4-130.

5. Jain S, Sharma M, Gupta R, et al. Multidrug resistant Shigella flexneri: a rare case of septicaemia in an infant. J Clin Diagn Res 2014;8(6):03-04. DOI: $10.7860 / J C D R / 2014 / 8434.4511$. 
6. Kamble R. Bacteraemia due to Shigella flexneri in an adult. Int J Curr Microbiol App Sci 2015;4(4):870-873. Available from: http://www. ijcmas.com.

7. Jauhari S, Nautiyal S, Singh S. Septicaemic invasion by Shigella flexneri. Sch J Med Case Rep 2017;5(7):434-435. DOI: 10.21276/sjmcr.

8. Sebhat $A E$, Endale $T$, Andargachew M, et al. A case of shigellosis with intractable septic shock and convulsions. Jpn J Infect Dis 2007;60(5):314-317. PMID: 17881877.

9. Lakxmikant C. Shigellosis with septicemia. J Assoc Phys India 1982;30:841. PMID: 7185828.

10. Yen JB, Chang KW, Wu TL, et al. Shigella flexneri sepsis in an Infant. Chang Gung Med J 2003;26(8):611-614. PMID: 14609044.

11. Saraswathi $K$, Anuradha $D E$, Jog A, et al. Shigella septicemia. Indian Pediatr 2002;39(8):777-779. PMID: 12196693.
12. Sharma S, Arora A. Shigella flexneri bacteremia in an adults. J Lab Physicians 2012;4(1):65-66. DOI: 10.4103/0974-2727.98682.

13. Kraybill EN, Controni G. Septicemia and enterocolitis due to Shigella sonnei in a newborn infant. Pediatrics 1968;42(3):529-31. PMID: 4877932.

14. Delgado M, Saenz C, Glasman P, et al. Shigella flexneri bacteremia in children: two case report and review of literature. Rev Chilena Infectol 2018;35(3):317-320. DOI: 10.4067/s0716-10182018000300317.

15. Ashkenazi S. Shigella infections: treatment and prevention in children. UpToDate 2014. Available from: http://www. uptodate. com/contents/ shigella infection-treatment and prevention-in-children.

16. Goren A, Freier S, Passwell JH. Lethal toxic encephalopathy due to childhood shigellosis in a developed country. Pediatrics 1992;89(6):1189-1193. PMID: 1594376. 